\title{
Optimized CEST cardiovascular magnetic resonance for assessment of metabolic activity in the heart
}

\author{
Zhengwei Zhou ${ }^{1,2}$, Christopher Nguyen ${ }^{1,3,4}$, Yuhua Chen ${ }^{1,2}$, Jaime L. Shaw ${ }^{1,2}, Z_{i x i n}$ Deng ${ }^{1,2}$, Yibin Xie',
} James Dawkins ${ }^{5}$, Eduardo Marbán ${ }^{5}$ and Debiao Li ${ }^{1,2,5^{*}}$

\begin{abstract}
Background: Previous studies have linked cardiac dysfunction to loss of metabolites in the creatine kinase system. Chemical exchange saturation transfer (CEST) is a promising metabolic cardiovascular magnetic resonance (CMR) imaging technique and has been applied in the heart for creatine mapping. However, current limitations include: (a) long scan time, (b) residual cardiac and respiratory motion, and (c) Bo field variations induced by respiratory motion. An improved CEST CMR technique was developed to address these problems.

Methods: Animals with chronic myocardial infarction $(N=15)$ were scanned using the proposed CEST CMR technique and a late gadolinium enhancement (LGE) sequence as reference. The major improvements of the CEST CMR technique are: (a) Images were acquired by single-shot FLASH, significantly increasing the scan efficiency. (b) All images were registered to reduce the residual motion. (c) The acquired Z-spectrum was analyzed using 3-pool-model Lorentzian-line fitting to generate CEST signal, reducing the impact of $\mathrm{B}_{0}$ field shifting due to respiratory motion. Feasibility of the technique was tested in a porcine model with chronic myocardial infarction. CEST signal was measured in the scar, border zone and remote myocardium. Initial studies were performed in one patient.

Results: In all animals, healthy remote myocardial CEST signal was elevated $(0.16 \pm 0.02)$ compared to infarct CEST signal $(0.09 \pm 0.02, P<0.001)$ and the border zone $(0.12 \pm 0.02, P<0.001)$. For both animal and patient studies, the hypointense regions in the CEST contrast maps closely match the bright areas in the LGE images.

Conclusions: The proposed CEST CMR technique was developed to address long scan times, respiratory and cardiac motion, and $B_{0}$ field variations. Lower CEST signal in bright region of the LGE image is consistent with the fact that myocardial infarction has reduced metabolic activity.
\end{abstract}

Keywords: MRI, Cardiac CEST, Creatine, Metabolic imaging, CEST CMR

\section{Background}

Adenosine triphosphate (ATP) is an essential energy source that governs myocardial contraction [1]. The creatine kinase (CK) system plays a vital role in the synthesis of myocardial ATP. ATP is generated from the conversion of phosphocreatine $(\mathrm{PCr})$ and adenosine diphosphate (ADP) to creatine $(\mathrm{Cr})$ catalyzed by $\mathrm{CK}$. Previous studies have linked cardiac dysfunction to the

\footnotetext{
* Correspondence: Debiao.Li@cshs.org

${ }^{1}$ Biomedical Imaging Research Institute, Cedars-Sinai Medical Center, 8700

Beverly Blvd. PACT Suite 400, Los Angeles, CA 90048, USA

${ }^{2}$ Department of Bioengineering, University of California Los Angeles, Los

Angeles, CA, USA

Full list of author information is available at the end of the article
}

loss of metabolites in the CK system [2-4]. The measurement of $\mathrm{Cr}$ or $\mathrm{PCr}$ can serve as a biomarker to investigate the metabolic change in the myocardium [5-9].

Chemical exchange saturation transfer (CEST) is an emerging MRI technique for metabolic imaging [10]. By saturating the solute protons, water signal will also drop because of the constant exchange between solute protons and water protons [11]. It has been shown that CEST can be used to map $\mathrm{Cr}$ distribution because of chemical transfer between its amine protons $\left(-\mathrm{NH}_{2}\right)$ and water protons. CEST can detect $\mathrm{Cr}$ separately from other CK metabolites because $\mathrm{Cr}$ protons alone have an intermediate transfer rate with water protons [12-14]. 
Compared to MR spectroscopy techniques, CEST yields better spatial resolution and higher sensitivity because of indirect detection via water protons $[14,15]$.

CEST has been applied in the heart for in vivo endogenous $\mathrm{Cr}$ imaging in animal studies [16-19]. Haris et al. was the first to show the feasibility of high-spatialresolution mapping of $\mathrm{Cr}$ using CEST cardiovascular magnetic resonance (CMR). However, the method poses some technical challenges which could potentially hinder the clinical translation of the CEST CMR technique: (a) long scan time ( $\sim 50 \mathrm{~min}$ per slice), (b) residual cardiac and respiratory motion, and (c) $B_{0}$ field variations mostly induced by respiratory motion.

In this study, we developed a clinically translatable CEST CMR technique with significantly reduced scan time ( $\sim 5 \mathrm{~min})$, improved motion registration and CEST signal analysis to address the aforementioned challenges. The proposed technique was then validated in chronic myocardial infarction porcine model using late gadolinium enhancement (LGE) as a reference. This animal model was chosen because it has been clearly shown that scar regions have reduced metabolic activity level compared to remote myocardium [20]. A repeatability study was performed in healthy subjects, and an initial patient study is presented.

\section{Methods}

\section{Animal study}

All animal-related procedures were approved by the Institutional Animal Care and Use Committee (IACUC) at Cedars-Sinai Medical Center. Myocardial infarction was induced in 15 Yucatan minipigs following the procedure described in a previous study [21]. Specifically, animals were sedated by intramuscular ketamine $20 \mathrm{mg} /$ $\mathrm{kg}$, acepromazine $0.25 \mathrm{mg} / \mathrm{kg}$ and atropine $0.05 \mathrm{mg} / \mathrm{kg}$, followed by $10 \mathrm{~mL}$ intravenous thiopental. Endotracheal intubation was then performed, and anesthesia maintained by ventilation with $1 \%$ to $2 \%$ isoflurane. Catheters were inserted through the left carotid artery. Coronary $\mathrm{X}$-ray angiography was performed to visualize the coronary arteries and identify the site for occlusion. An anteroseptal myocardial infarction was induced by inflation of an angioplasty balloon in the mid-left anterior descending artery to cause coronary occlusion for $2.5 \mathrm{~h}$. Finally, the animals were taken to the post-op recovery room. All imaging was performed eight weeks after the infarction.

Animal study $(N=15)$ was performed on a 3 Tesla clinical scanner (Magnetom Verio; Siemens Healthineers, Erlangen, Germany) using a 12-channel phase array coil for data acquisition. Throughout the imaging procedures, anesthesia was maintained with isoflurane $(1-3.5 \%)$.
The imaging protocol included balanced steady state free precession (bSSFP) cine, CEST CMR and LGE. bSSFP cine was performed to localize the quiescent period of the cardiac cycle $\left(1.4 \times 1.4 \times 6 \mathrm{~mm}^{3}\right.$; electrocardiogram-gated; 35 cardiac phases; $\mathrm{TR} / \mathrm{TE}=3.2 / 1.6 \mathrm{~ms}$; flip angle $50^{\circ}$ ). LGE imaging was completed $15 \mathrm{~min}$ after the contrast agent injection $(0.1 \mathrm{mmol} / \mathrm{kg}$, gadobutrol, Gadovist, Bayer Healthcare, Berline, German) using phase sensitive inversion recovery FLASH $\left(1.3 \times 1.3 \times 6 \mathrm{~mm}^{3}\right.$; TR/TE/TI = $362 / 1.5 / 335 \mathrm{~ms}$; flip angle $20^{\circ}$ ). All imaging was performed in the short axis plane at three mid-ventricular slice locations of the left ventricle. Breath hold was controlled by a ventilator in cine imaging and LGE imaging.

CEST CMR scans were performed before the contrast injection using FLASH readout (resolution $2.1 \times 2.1 \mathrm{~mm}^{2}$; slice thickness $6-8 \mathrm{~mm}$; TR/TE $=4000 / 1.5 \mathrm{~ms}$; flip angle $\left.12^{\circ}\right)$. Figure 1 shows the pulse sequence diagram of the proposed CEST CMR technique. Electrocardiogram triggering and navigator gating were used to reduce the effects of cardiac and respiratory motion. Each image was acquired by single-shot FLASH ( 200 ms readout block placed in quiescent period). Thirty-three images were collected at different saturation frequency offsets ranging from $-4.8 \mathrm{ppm}$ to $4.8 \mathrm{ppm}$ with a step size of $0.3 \mathrm{ppm}$. The CEST preparation module consists of five Gaussian-shaped pulses with $2700^{\circ}$ flip angle and $30 \mathrm{~ms}$ duration at a duty cycle of $50 \%$ (the equivalent $B_{1}$ power is $3.76 \mathrm{uT}$ ). The spoiler gradient was altered in different directions to crush the residual transverse magnetization. An additional image was acquired without CEST saturation for normalization reference. The scan time for each slice was approximately 5 min with $40 \%$ navigator efficiency.

CEST CMR technique was optimized in the following aspects: (a) Images were acquired by single-shot FLASH instead of segmented acquisition, significantly increasing

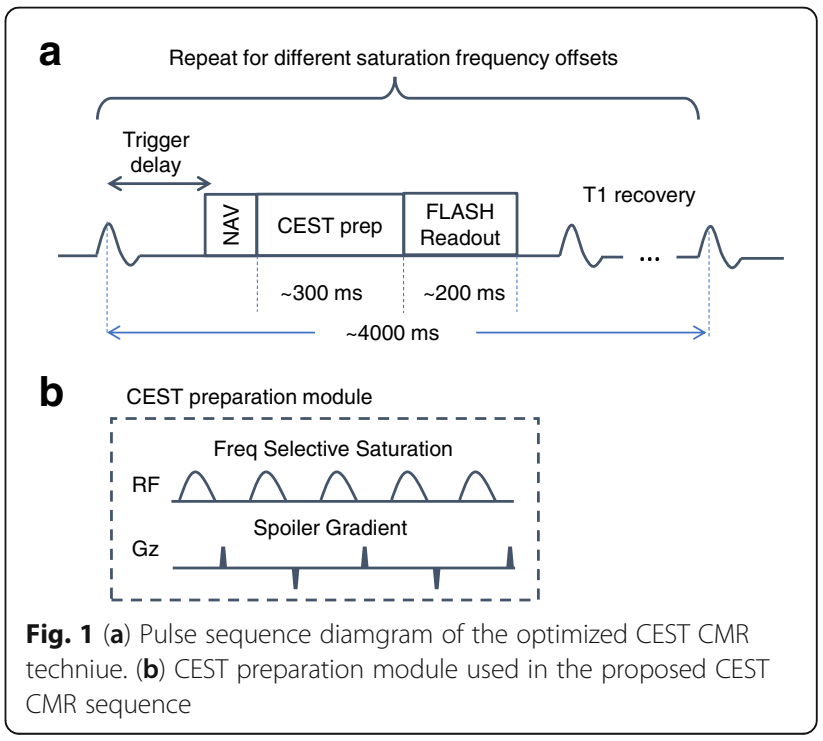


the scan efficiency. (b) All images were registered to reduce the residual left ventricle wall motion (up to $4 \mathrm{~mm}$ myocardial displacement) using Medical Imaging Registration Toolbox (MIRT) [22]. (c) The acquired Zspectrum was fitted to the Lorentzian-shaped 3-poolmodel to increase the reliability of the generated CEST contrast map by reducing the impact of signal fluctuation from $\mathrm{B}_{0}$ field shifting introduced by respiratory motion.

\section{Healthy subject study}

CEST CMR in healthy subjects $(n=14)$ was performed on a $3 \mathrm{~T}$ clinical scanner (Magnetom Verio; Siemens Healthineers) using a 32-channel phase array coil for data acquisition. For the healthy subject and patient imaging studies, the protocol was approved by Institutional Review Board at Cedars-Sinai Medical Center and written informed consent was obtained from each subject before the exam. For each healthy subject, repeatability of the proposed CEST CMR method was evaluated in two mid-ventricular slices by repeating the scan twice in the same imaging position.

The parameters of CEST CMR sequence used in healthy subject study were similar compared to the animal study. Each image was acquired by single-shot FLASH (Field-of-view: $350 \times 280 \mathrm{~mm}^{2}$; spatial resolution: $2.7 \times 2.2 \times 8.0 \mathrm{~mm}^{3} ; \quad \mathrm{TR} / \mathrm{TE}=4000 / 1.45 \mathrm{~ms}$; matrix size: $160 \times 104$; iPAT: 2 ; flip angle: $12^{\circ}$; acquisition window: $163 \mathrm{~ms}$ ). 3 Gaussian-shaped pulses with flip angle of $2700^{\circ}$ and pulse duration of $80 \mathrm{~ms}$ were used in the CEST preparation module. The interpulse delay was $80 \mathrm{~ms}$. Equivalent RF irradiation power is $1.90 \mathrm{uT}$. CEST saturation frequency offsets range from $-3.6 \mathrm{ppm}$ to $3.6 \mathrm{ppm}$ with a step size $0.3 \mathrm{ppm}$. An additional image was acquired without CEST saturation for normalization reference. The scan time for each slice was approximately 5 min with $30 \%$ navigator efficiency.

\section{Image analysis}

Post processing was performed with custom-written programs in Matlab (The Mathworks, Natick, Massachusetts, USA). For each pixel, the Z-spectrum was generated as the signal intensity of each image acquired at different saturation frequency offsets normalized by the reference image. By using 3-pool-model Lorentzian-line fitting, the acquired Z-spectrum was separated into CEST, direct water saturation (DWS), and conventional magnetization transfer (MT), three major effects in saturation experiments with aqueous solutions $[16,23]$. The center of the DWS curve is the central water frequency, which represents $B_{0}$ field information. The distance between the center of the CEST curve and DWS curve is constantly $+1.8 \mathrm{ppm}$, which is the resonant frequency of amine protons. CEST signal is defined as the amplitude of the fitted CEST curve.
CEST maps were generated using pixel-by-pixel $\mathrm{Z}$-spectrum fitting in the myocardium. In the animal study, regions of interest (ROIs) were placed in the scar region, remote myocardium and border zone adjacent to the infarcted myocardium using LGE as reference. The average CEST signal was determined in scar, border zone and healthy remote myocardium, for each subject. Comparisons were performed using repeated measures one-way ANOVA analysis followed by Tukey's multiple comparisons test in GraphPad Prism 6 (Graph-Pad Software, La Jolla, California, USA). A two-tailed value of $p<0.05$ was considered to be statistically significant. In the healthy subject studies, the average CEST signal was calculated in the entire myocardium ("global") and in the septum. The repeatability was evaluated by performing Bland-Altman plot in GraphPad Prism 6 (GraphPad Software).

\section{Patient study}

One patient who was referred for LGE CMR exam for evaluation of chronic myocardial infarction was studied using the proposed CEST CMR sequence with the same settings/parameters as the volunteer studies. LGE imaging was completed 15 min after the contrast agent injection $(0.1 \mathrm{mmol} / \mathrm{kg}$, gadobutrol, Gadovist, Bayer Inc.) using phase sensitive inversion recovery FLASH $(1.4 \times$ $1.4 \times 8 \mathrm{~mm}^{3} ; \mathrm{TR} / \mathrm{TE} / \mathrm{TI}=330 / 1.6 / 300 \mathrm{~ms}$; flip angle $\left.20^{\circ}\right)$.

\section{Results}

\section{Animal study}

In total, the CEST CMR scans were performed in 15 animals. The heart rates of the animals were in the range of $100-120$ beats/min during the CMR scan.

To separate the CEST signal from MT and DWS effects, we used 3-pool-model fitting to analyze the Z-spectrum (Fig. 2). The peak of the CEST curve is defined as CEST signal, which is used in the following quantitative analysis. Comparing the $\mathrm{Z}$-spectrum analysis curves in the scar with those in the healthy myocardium, it is clear that CEST signal is lower in the scar region.

Figure 3 shows typical CEST contrast maps and corresponding LGE images in three slice locations. The infarcted regions have lower CEST signal than healthy remote myocardium. Spatially, the hypointense regions in the CEST contrast maps closely match the bright areas in LGE images.

In all animals, infarct CEST signal $(0.09 \pm 0.02)$ is significantly reduced compared to the CEST signal found in the healthy remote myocardium $(0.16 \pm 0.02), p<0.001$, as shown in Fig. 4. The CEST signal in the border zone $(0.12 \pm 0.02)$ was also reduced compared to the remote myocardium, $p<0.001$, while it was elevated compared to the infarcted tissue, $p<0.001$. 

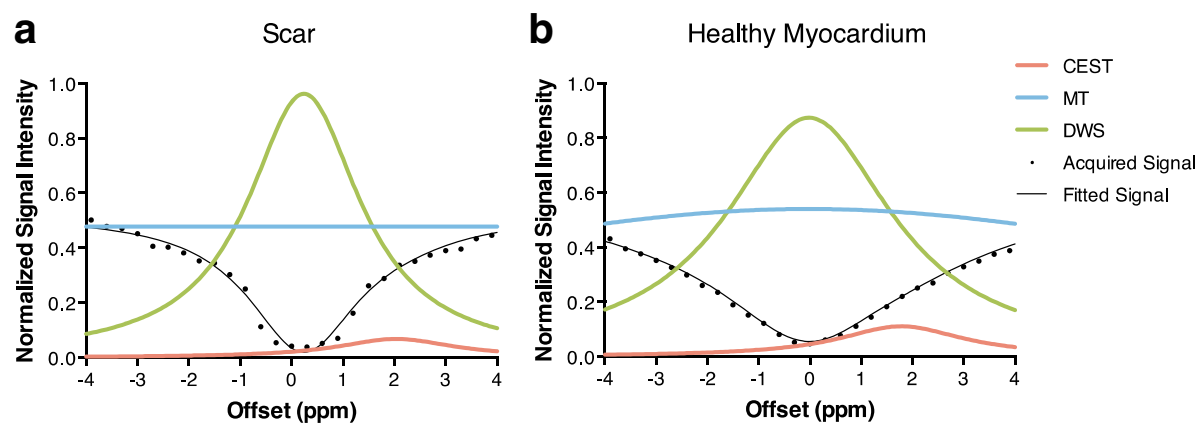

Fig. 2 Z-spectrum of (a) scar region and (b) healthy myocardium. By using the 3-pool-model fitting, Z-spectrum (black) was separated into CEST curve (pink), MT curve (blue) and DWS curve (green). The center of DWS curve represents the resonant water frequency ( $B_{0}$ field). The peak of CEST curve was defined as the CEST signal. It is clear that the healthy myocardium has higher CEST signal than scar, which suggests more Cr distribution in the healthy myocardium

\section{Healthy subject study}

Fourteen healthy subjects (10 male, $39 \pm 13$ years, BMI $24 \pm 4)$ were included in the studies. The average scan time of each slice was $4.8 \pm 2.8 \mathrm{~min}$.

The Bland-Altman plots of global CEST signal and septal CEST signal were shown in Fig. 5a and b, respectively. The $95 \%$ limits of agreement of the global CEST signal were -0.07 to 0.06 , while the $95 \%$ limits of agreement of the septal CEST signal were -0.06 to 0.05 .

\section{Patient study}

The CEST map and the corresponding LGE image of the patient study were shown in Fig. 6 . The hypointense region in the CEST maps is consistent with the scar region shown on the LGE image.

\section{Discussion}

In this work, we developed a CEST CMR technique with significantly reduced scan time ( $\sim \mathrm{min})$, improved motion registration and CEST signal analysis to show the feasibility of the proposed technique in detecting $\mathrm{Cr}$ distribution in the myocardium in a clinically feasible scan time. The animal study showed decreased CEST signal in infarcted region compared to remote myocardium, which is consistent with previous finding [16]. The repeatability healthy subject studies and the preliminary patient result also suggest initial feasibility of the proposed CEST CMR technique.

As CK metabolites are essential in providing energy for myocardial contraction, cardiac metabolic impairment is now considered as a cause, rather than a result of cardiac diseases [24]. Detection of CK metabolites
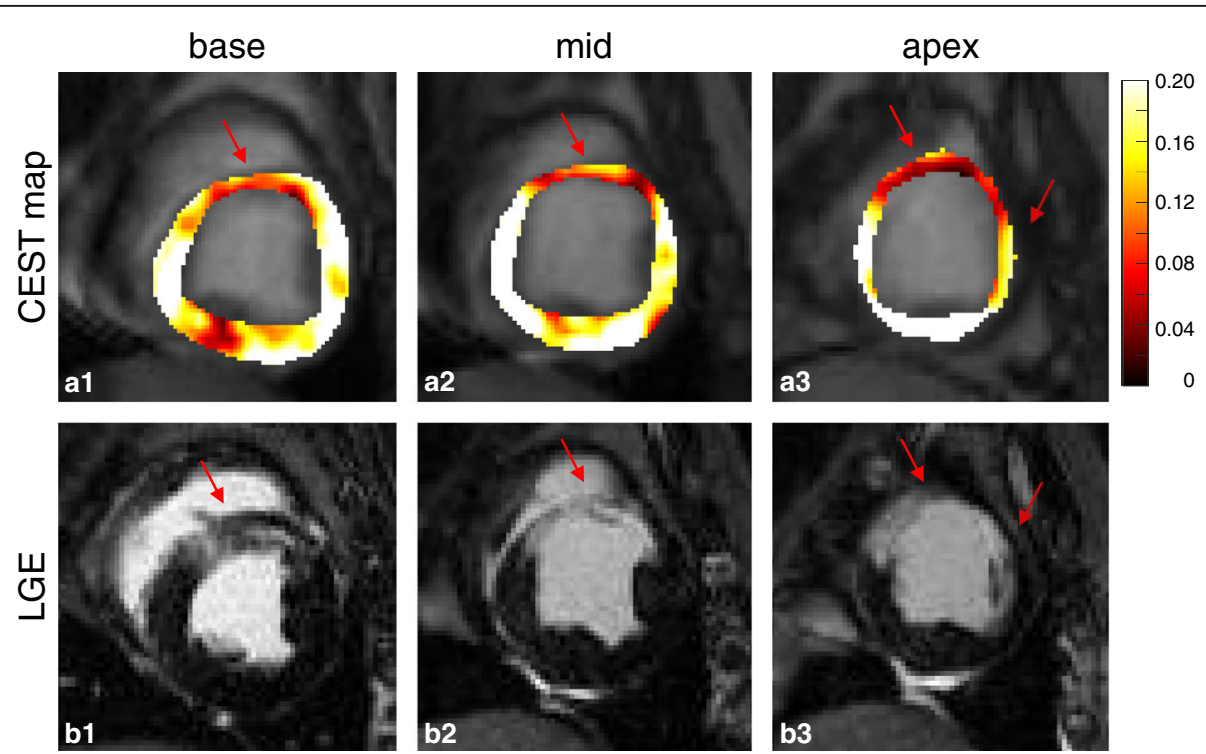

Fig. 3 (a) CEST maps and (b) corresponding LGE images of one representative subject in three slice locations. The hypointense regions (arrows) in the CEST maps match the LGE positive regions (arrows) 


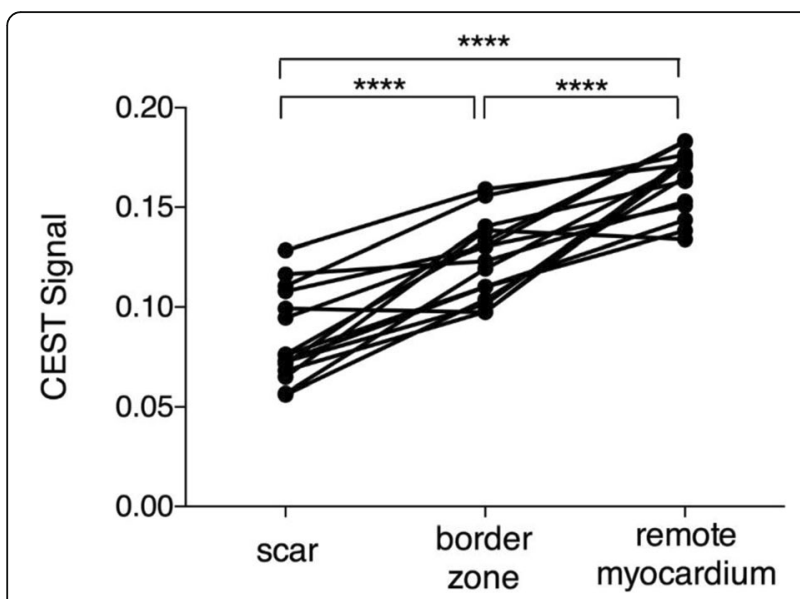

Fig. 4 CEST signal in the scar $(0.09 \pm 0.02)$ is significantly reduced compared to healthy remote myocardium $(0.16 \pm 0.02), p<0.001$. CEST signal in the border zone $(0.12 \pm 0.02)$ was also reduced compared to remote myocardium, $p<0.001$

such as creatine can potentially help the clinical diagnosis and treatment of cardiovascular diseases. It can be used to further understand the underlying mechanisms of metabolic change processes in these diseases as well as to evaluate therapy efficiency and guide therapy optimization.

In this study, we compared the CEST signal in the scar, border zone and remote myocardium. As expected, the CEST signal in the scar was significantly reduced compared to the CEST signal in the remote myocardium. This is consistent with the previous findings that the infarcted tissue has reduced metabolic activity [20]. More interestingly, the CEST signal in the border zone was also significantly reduced compared to the remote myocardium. This potentially suggests that the border zone might also have reduced myocardial metabolic activity. However, a more comprehensive study needs to be performed to further demonstrate this point.

Cardiac MR Spectroscopy (CMRS) is also capable of detecting the activity of CK metabolites in the myocardium. $\mathrm{PCr}$ and ATP can be detected as two peaks in ${ }^{31} \mathrm{P}$ spectrum. The ratio of PCr over ATP can serve as an indicator of myocardial energy metabolism $[5,25]$. In ${ }^{1} \mathrm{H}$ spectrum, $\mathrm{Cr}$ and $\mathrm{PCr}$ have the same chemical shift and only their combined total amount can be measured $[6,26]$.
However, these techniques have not gained common application over the years because of limitations such as low spatial resolution, lengthy scan time, and complicated acquisition protocol. ${ }^{31} \mathrm{P}$ CMRS also requires additional hardware, which limits its availability. All of these issues hinder the clinical translation of CMRS approaches. However, they can be potentially addressed by CEST CMR techniques. In this study, we have shown the feasibility of CEST CMR technique with typical CMR spatial resolution and clinically acceptable scan time. CEST CMR is also more sensitive compared to CMRS techniques because the signal is indirectly detected from the water pool. Without any special requirement for hardware or technician training, this technique can be easily translated into clinical studies.

The CEST CMR sequence we proposed in this study is significantly faster than the previous technique [16]. One of the major reasons is that we used single-shot FLASH readout instead of segmented readout. In addition to its contribution to speed acceleration, single-shot readout also minimizes the respiratory motion within the acquisition of each CEST-weighted image at different saturation frequency offsets. This reduces the motion artifacts and $\mathrm{B}_{0}$ field changes within the acquisition of each single image. It should be noted, though, when the subject has a relatively high heart rate and short quiescent period, the image acquired using single-shot readout will be blurrier. More advanced image reconstruction methods can be used in the future to shorten the acquisition period.

It is known that the heart is one of the most difficult organs for imaging because of constant cardiac motion and respiratory motion. In CEST CMR scans, in addition to the motion within the acquisition of one image that leads to blurriness and the misregistration between different images that causes error in pixel-by-pixel mapping, we are also facing $\mathrm{B}_{0}$ field variations caused by residual motion. Respiratory phase mismatch, even within the navigator acceptance window, leads to $\mathrm{B}_{0}$ field variations in the myocardium for images acquired with different saturation frequency offsets. Because of the relative position of heart (tissue) and lung (air) in the lateral wall, the $\mathrm{B}_{0}$ field is changing rapidly in the heart, especially the lateral wall region. In this study, 3-pool-model fitting is used to generate
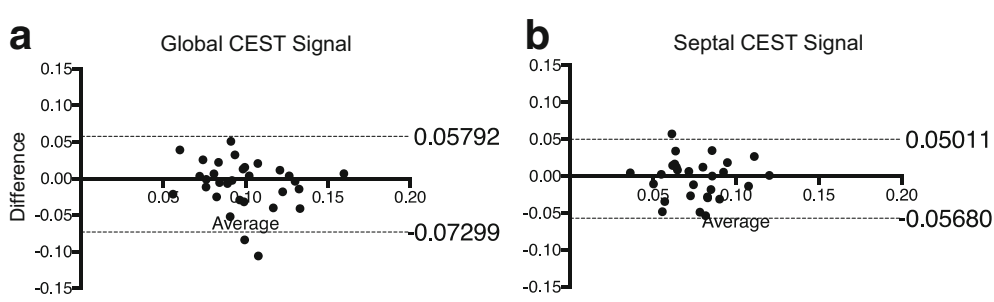

Fig. 5 Bland-Altman plots of (a) global CEST signal and (b) septal CEST signal. The dashed lines represent 95\% limits of agreement 

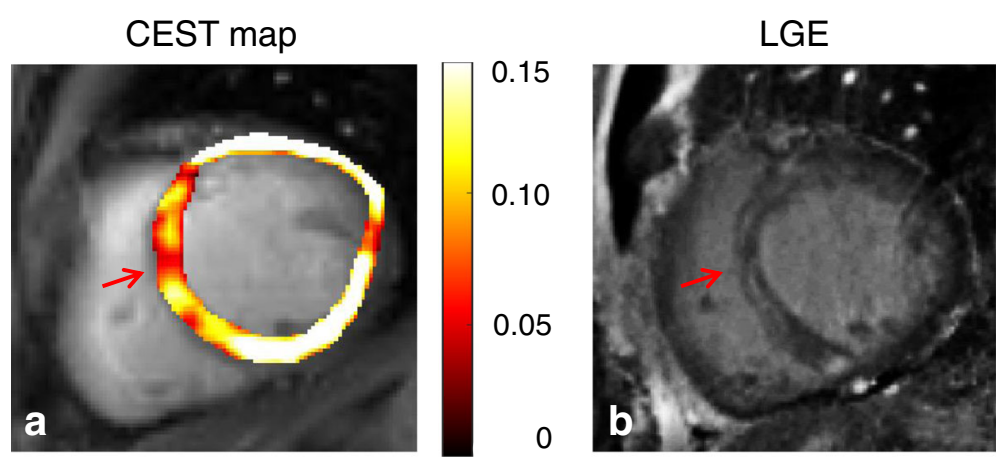

Fig. 6 (a) CEST map and (b) corresponding LGE image in the patient with a chronic myocardial infarction

CEST signal so the $\mathrm{B}_{0}$ field variations can be treated as noise. However, this method can address this issue only to certain extent. Therefore, sometimes inhomogeneity is observed in the CEST map (Fig. 3), especially in the lateral wall region. This issue can be potentially addressed using advanced shimming coil design so the $\mathrm{B}_{0}$ field over the myocardium can be more homogeneous. Another potential method could be incorporating additional $\mathrm{B}_{0}$ field information in the data acquisition.

This study shows the feasibility of CEST CMR technique in healthy subjects and animal studies. Preliminary patient result is also presented. However, to further demonstrate the utility of CEST CMR technique in patients, more comprehensive studies need to be performed. The animal model used in this study only yielded transmural infarcts. Non-transmural infarcts are typically smaller in size and may be more difficult to detect. Whether the spatial resolution and sensitivity of the current technique is enough for non-transmural infarcts requires more studies. Additionally, the data acquired in this study were limited to the left anterior descending territory. Further studies will be needed to evaluate the performance of CEST CMR at different arterial territories.

In this study, we applied CEST CMR in the wellstudied chronic infarction model using LGE as reference. However, CEST CMR should not be seen as a potential replacement for LGE for scar quantification. As a supplement, CEST CMR provides the unique metabolic information which could potentially be used to study the undergoing mechanisms in cardiac dysfunctions. However, more comprehensive studies still need to be performed to demonstrate the utility of CEST CMR. The results in this study show that the infarct region has about $50 \%$ drop in CEST signal compared to healthy remote myocardium. In some types of cardiac dysfunctions which have mild metabolic impairment, the difference of signal could be smaller. Further investigation is needed to evaluate whether CEST CMR technique has the reproducibility and sensitivity to detect cardiac dysfunctions with mild metabolic abnormalities. Histopathology studies are also needed to demonstrate the CEST signal change is correlated to creatine distribution in the myocardium.

\section{Conclusion}

We developed a clinically feasible CEST CMR technique that is significantly faster than the previous approach. In a chronic myocardial infarction pig model, we demonstrated the proposed CEST CMR technique can discern differences in the CEST signal between infarct and remote myocardium. This study shows the feasibility of CEST CMR technique in healthy subjects, animal studies, and a preliminary patient study.

\section{Abbreviations}

ADP: Adenosine diphosphate; ATP: Adenosine triphosphate; bSSFP: Balanced steady state free precession; CEST: Chemical exchange saturation transfer; CK: Creatine kinase; CMR: Cardiovascular magnetic resonance; Cr: Creatine; DWS: Direct water saturation; FLASH: Fast low angle shot; LGE: Late gadolinium enhancement; MT: Magnetization transfer; PCr: Phosphocreatine; ROI: Region of interest

\section{Acknowledgements}

The authors thank Edward Gill, Laura G. Smith, Hernan Rios and Richard Tang for help in conducting the imaging experiments.

\section{Funding}

This work was supported in part by 2016 SCMR Seed Grant.

\section{Availability of data and materials}

The datasets acquired and/or analyzed during the current study are not publicly available but are available from the corresponding author on reasonable request.

\section{Authors' contributions}

ZZ and DL conceived the study. ZZ implemented the method, performed the data acquisition and drafted the manuscript. $Y C$ assisted with the motion registration. CN, JS, ZD, YX and DL assisted with the study design. CN, JS, ZD, $Y X, M B$ and $D L$ provided critical review of the manuscript. JD developed the animal model. MB supported the animal studies. All authors read and approved the final manuscript.

Ethics approval and consent to participate

For the animal studies, the protocol was approved by the local Institutional Animal Care and Usage Committee (IACUC) at Cedars-Sinai Medical Center.

For the volunteer and patient studies, the protocol was approved by the 
Institutional Review Board (IRB). Written informed consent was obtained from each subject before the exam.

\section{Competing interests}

The authors declare that they have no competing interests.

\section{Publisher's Note}

Springer Nature remains neutral with regard to jurisdictional claims in published maps and institutional affiliations.

\section{Author details}

'Biomedical Imaging Research Institute, Cedars-Sinai Medical Center, 8700 Beverly Blvd. PACT Suite 400, Los Angeles, CA 90048, USA. ²Department of Bioengineering, University of California Los Angeles, Los Angeles, CA, USA. ${ }^{3}$ Cardiovascular Research Center, Massachusetts General Hospital, Charlestown, MA, USA. ${ }^{4}$ Harvard Medical School, Boston, MA, USA. ${ }^{5}$ Heart Institute, Cedars-Sinai Medical Center, Los Angeles, CA, USA.

Received: 4 April 2017 Accepted: 20 November 2017

\section{Published online: 30 November 2017}

\section{References}

1. Weiss RG and Maslov M. Normal myocardial metabolism: fueling cardiac contraction. Adv Stud Med. 2004;4:S457-S463.

2. Nascimben L, Ingwall JS, Pauletto P, Friedrich J, Gwathmey JK, Saks V, Pessina AC, Allen PD. Creatine kinase system in failing and nonfailing human myocardium. Circulation. 1996;94:1894-901.

3. Ingwall JS, Atkinson DE, Clarke K, Fetters JK. Energetic correlates of cardiac failure: changes in the creatine kinase system in the failing myocardium. Eur Heart J. 1990;11:108-15.

4. Ventura Clapier R, Garnier A, Veksler V. Energy metabolism in heart failure. J Physiol. 2004:555:1-13.

5. Bottomley PA, Weiss RG. Non-invasive magnetic-resonance detection of creatine depletion in non-viable infarcted myocardium. Lancet. 1998, 351:714-8.

6. Nakae I, Mitsunami K, Omura T, et al. Proton magnetic resonance spectroscopy can detect creatine depletion associated with the progression of heart failure in cardiomyopathy. J Am Coll Cardiol. 2003;42:1587-93.

7. Neubauer S, Horn M, Cramer M, et al. Myocardial phosphocreatine-to-ATP ratio is a predictor of mortality in patients with dilated cardiomyopathy. Circulation. 1997:96:2190-6.

8. Dawson DK, Neil CJ, Henning A, Cameron D, Jagpal B, Bruce M, Horowitz J, Frenneaux MP. Tako-Tsubo cardiomyopathy: a heart stressed out of energy? JACC Cardiovasc Imaging. 2015;8:985-7.

9. Beer M, Seyfarth T, Sandstede J, Landschütz W, Lipke C, Köstler H, Kienlin v M, Harre K, Hahn D, Neubauer S. Absolute concentrations of high-energy phosphate metabolites in normal, hypertrophied, and failing human myocardium measured noninvasively with (31)P-SLOOP magnetic resonance spectroscopy. J Am Coll Cardiol. 2002;40:1267-74.

10. Ward KM, Aletras AH, Balaban RSA. New class of contrast agents for MRI based on proton chemical exchange dependent saturation transfer (CEST) J Magn Reson. 2000;143:79-87.

11. van Zijl PCM, Yadav NN. Chemical exchange saturation transfer (CEST): what is in a name and what isn't? Magn Reson Med. 2011:65:927-48.

12. Haris M, Nanga RPR, Singh A, Cai K, Kogan F, Hariharan H, Reddy R. Exchange rates of creatine kinase metabolites: feasibility of imaging creatine by chemical exchange saturation transfer MRI. NMR Biomed. 2012;25:1305-9.

13. Kogan F, Haris M, Debrosse C, Singh A, Nanga RP, Cai K, Hariharan H, Reddy R. Vivo chemical exchange saturation transfer imaging of creatine (CrCEST) in skeletal muscle at 3T. J Magn Reson Imaging. 2014;40:596-602.

14. Kogan F, Haris M, Singh A, Cai K, Debrosse C, Nanga RPR, Hariharan H, Reddy R. Method for high-resolution imaging of creatine in vivo using chemical exchange saturation transfer. Magn Reson Med. 2014;71:164-72.

15. Zhou J, Payen J-F, Wilson DA, Traystman RJ, van Zijl PCM. Using the amide proton signals of intracellular proteins and peptides to detect pH effects in MRI. Nat Med. 2003;9:1085-90.

16. Haris $\mathrm{M}$, Singh $\mathrm{A}$, Cai $\mathrm{K}$, et al. A technique for in vivo mapping of myocardial creatine kinase metabolism. Nat Med. 2014;20:209-14.

17. Vandsburger M, Vandoorne K, Oren R, Leftin A, Mpofu S, Delli Castelli D, Aime S, Neeman M. Cardio-chemical exchange saturation transfer magnetic resonance imaging reveals molecular signatures of endogenous fibrosis and exogenous contrast media. Circ Cardiovasc Imaging. 2015:8:e002180.

18. Pumphrey A, Yang Z, Ye S, et al. Advanced cardiac chemical exchange saturation transfer (cardioCEST) MRI for in vivo cell tracking and metabolic imaging. NMR Biomed. 2016;29:74-83.

19. Pumphrey AL, Ye S, Yang Z, Simkin J, Gensel JC, Abdel-Latif A, Vandsburger $\mathrm{MH}$. Cardiac chemical exchange saturation transfer MR imaging tracking of cell survival or rejection in mouse models of cell therapy. Radiology. 2017; 282:131-38.

20. Sanbe A, Tanonaka K, Hanaoka Y, Katoh T, Takeo S. Regional energy metabolism of failing hearts following myocardial infarction. J Mol Cell Cardiol. 1993;25:995-1013.

21. Lee S-T, White AJ, Matsushita S, et al. Intramyocardial injection of autologous cardiospheres or cardiosphere-derived cells preserves function and minimizes adverse ventricular remodeling in pigs with heart failure post-myocardial infarction. J Am Coll Cardiol. 2011;57:455-65.

22. Myronenko A. Xubo song. Intensity-based image registration by minimizing residual complexity. Medical Imaging, IEEE Transactions on. 2010;29:1882-91.

23. Zaiss $M$, Schmitt B, Bachert P. Quantitative separation of CEST effect from magnetization transfer and spillover effects by Lorentzian-line-fit analysis of z-spectra. J Magn Reson. 2011;211:149-55.

24. Neubauer S. The failing heart - an engine out of fuel. N Engl J Med. 2007; 356:1140-51.

25. Bottomley PA, Weiss RG. Noninvasive localized MR quantification of creatine kinase metabolites in normal and infarcted canine myocardium. Radiology. 2001:219:411-8

26. Bottomley PA, Lee $Y$, Weiss RG. Total creatine in muscle: imaging and quantification with proton MR spectroscopy. Radiology. 1997;204:403-10.

\section{Submit your next manuscript to BioMed Central and we will help you at every step:}

- We accept pre-submission inquiries

- Our selector tool helps you to find the most relevant journal

- We provide round the clock customer support

- Convenient online submission

- Thorough peer review

- Inclusion in PubMed and all major indexing services

- Maximum visibility for your research

Submit your manuscript at www.biomedcentral.com/submit
) Biomed Central 